\title{
The Frequency-Dependent Directivity of a Planar Fabry-Perot Polymer Film Ultrasound Sensor
}

\author{
Benjamin T. Cox and Paul C. Beard
}

\begin{abstract}
A model of the frequency-dependent directivity of a planar, optically-addressed, Fabry-Perot (FP), polymer film ultrasound sensor is described and validated against experimental directivity measurements made over a frequency range of 1 to $15 \mathrm{MHz}$ and angles from normal incidence to $80^{\circ}$. The model may be used, for example, as a predictive tool to improve sensor design, or to provide a noise-free response function that could be deconvolved from sound-field measurements in order to improve accuracy in high-frequency metrology and imaging applications. The specific question of whether effective element sizes as small as the optical-diffraction limit can be achieved was investigated. For a polymer film sensor with a FP cavity of thickness $d$, the minimum effective element radius was found to be about $0.9 d$, and that an illumination spot radius of less than $d / 4$ is required to achieve it.
\end{abstract}

\section{INTRODUCTION}

$\mathrm{T}$ HE use of thin film Fabry-Perot interferometers (FPI) for the detection of ultrasound in liquids at megahertz frequencies provides a practical alternative to broadband piezoelectric receivers for a variety of measurement and imaging applications [1]-[14]. The sensing element comprises a thin film spacer $(<50 \mu \mathrm{m})$ sandwiched between optically reflective coatings and overlaying a transparent acoustically thick substrate. It is termed a planar sensing structure when the lateral dimensions are on a centimeter scale and thus large compared to an acoustic wavelength at megahertz frequencies, in contrast to configurations of limited, lateral extent, such as when the sensor is formed on the tip of an optical fiber [2], [3], [4].

The sensor is typically interrogated by illuminating it with the free-space output of a laser and directing the reflected beam onto a photodiode. An incident ultrasound wave modulates the optical thickness of the sensing structure. This produces a corresponding optical phase shift between the light reflected from its two faces and, therefore, a modulation in the power of the reflected beam. Assuming the phase bias or working point of the FPI is set to the maximum slope of the FPI intensity-phase transfer func-

Manuscript received March 13, 2006; accepted August 7, 2006. This work was supported by the Engineering and Physical Sciences Research Council, UK.

The authors are with the Department of Medical Physics and Bioengineering, University College London, London, WC1E 6BT, UK. (e-mail: bencox@mpb.ucl.ac.uk).

Digital Object Identifier 10.1109/TUFFC.2007.253 tion (by adjusting the wavelength or angle of incidence of the interrogating laser beam), the sensor provides a linear output for acoustically induced phase shifts.

In terms of acoustic performance, the fundamental advantage of the concept is the ability to provide highdetection sensitivities, comparable to broadband piezoelectric PVDF receivers, but with significantly smaller element sizes. The latter is determined by the dimensions of the optical field that addresses the sensor and, therefore, can be reduced, in principle, to the optical diffraction limit of a few microns. This offers the unique prospect of realizing the small element sizes required to provide an omnidirectional response at frequencies in the tens of megahertz range.

Several variants of this approach, distinguished by the materials and methods used to fabricate the FPI and the means to optically address it, have been demonstrated. One fabrication method involves sputtering a multilayer dielectric structure comprising two high reflectivity dielectric mirrors on either side of a $\mathrm{SiO}_{2}$ spacer onto a glass substrate [5]. The small thickness of the FPI formed using this method (typically a few microns) offers the prospect of bandwidths extending to several hundred megahertz. Another approach has been to use a thicker spacer (typically tens of microns) fabricated using a polymer film to provide bandwidths in the tens of megahertz range [6]-[8]. For both types of sensor, a variety of optical addressing schemes have been used, the simplest being to illuminate with a focused [5] or collimated laser beam [6] to form a single-element receiver. More elaborate schemes that address different points on the sensor surface, either sequentially or in parallel, enabling the spatial distribution of ultrasound fields to be measured also have been described. These methods include optically scanning a focused laser beam across the sensor [9]-[11] or illuminating with a large area laser beam and mapping the reflected sensor output beam using a mechanically scanned photodiode [12] or an array of detectors such as a CCD camera [10], [13]. The ability of these systems to map ultrasound fields with high spatial-temporal resolution has led to a variety of field characterization and imaging applications, including mapping the output of broadband ultrasound transducers [10][12], high resolution photoacoustic imaging of biological tissues [8], [14], and transmission ultrasound imaging [12].

Although the practical application of these sensors has been successfully demonstrated, predictive theoreti- 
cal models that fully describe their acoustic performance in terms of frequency response and directivity have yet to be developed. Previously, an experimentally validated analytic model of the frequency response of a planar FabryPerot (FP) polymer film sensor has been described [6]. However, this model provided the sensor response due to normally incident plane waves only. In this paper, a more general analytic model is developed that describes the complex, frequency-dependent, directional response (magnitude and phase) of the sensor. As well as providing insight into the physical mechanisms that underlie the acoustic characteristics of the sensor, such a model provides a valuable simulation tool for developing optimized sensor designs by careful selection of the geometric and material parameters. Additionally, it can be used to deconvolve for the directional response of the sensor to reduce spatial averaging errors in quantitative ultrasound metrology and blurring and artefacts in imaging applications.

One specific application of the model that is of widespread significance to the practical application of the technique, and a focus of this paper, is its use to determine the limits to the commonly held assumption that the element size is defined by the dimensions of the optical field that addresses the sensor. Although intuitively reasonable for a laser spot radius much larger than the FPI thickness, there will be some limit to this assumption as the spot radius is reduced, and the question as to whether it really is possible to obtain element sizes down to the optical diffraction limit of a few microns (as one might hope for) remains unanswered. Because the directional response is directly related to element radius, this limit can be determined by using the model to compute the directivity and comparing it to that of an ideal ultrasound receiver to obtain an effective element radius as a function of the illumination spot radius. It then should be possible to identify the smallest element size that can realistically be achieved for a given sensor geometry and material properties. This has important implications for the design of the optical interrogation instrumentation. For example, there is little to be gained in undertaking the nontrivial task of designing an optical system that provides for truly optical diffraction limited spot sizes, if the effective element radius for a specific sensor configuration is significantly larger.

Although the model described in this paper is generally applicable to planar FPI ultrasound sensors, we consider by way of an example, the specific case of those that use a polymer film spacer. Thus in Section II, the sensor fabrication, typical material and geometric properties of this configuration, are outlined. The model and its experimental validation are described in Sections III and IV. Two applications of the model are discussed in Section V. First, it is used to demonstrate how the directional response can be improved by appropriate selection of the material properties of the backing substrate. Second, to investigate the relationship between the dimensions of the region of the sensor that is optically addressed and the effective element radius in order to determine the minimum attainable value of the latter.

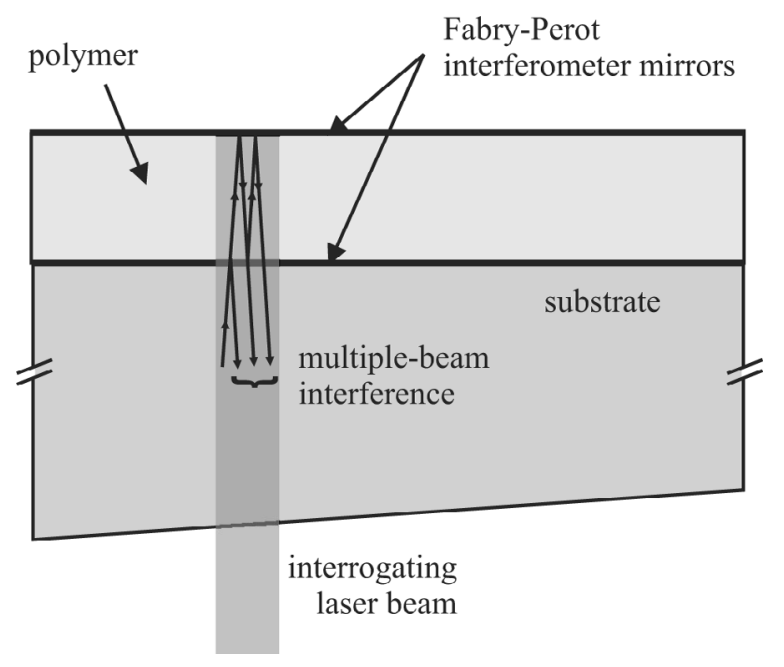

Fig. 1. A FP ultrasound sensor comprising a polymer layer (10$50 \mu \mathrm{m})$ with two thin reflective coatings, forming the FP interferometer, overlying a solid glass or polymer substrate $(1-2 \mathrm{~cm}$ thick). The substrate has an angled back-face to prevent optical reflections within it acting as a second interferometer. The shape and size of the acoustically sensitive region is determined by the profile of the interrogating laser beam and the acoustic properties of the materials. The width is not to scale and is typically of centimeter order.

\section{Fabry-Perot Polymer Film Ultrasound SEnsor}

A schematic of a FP polymer film sensor is shown in Fig. 1. It consists of a layer of polymer sandwiched between two thin, optically reflective coatings and overlying a transparent solid substrate, typically several centimeters thick. The polymer layer is typically $10-50 \mu \mathrm{m}$ thick and forms the interior of the FP cavity, with the reflective layers forming the mirrors of the cavity. Several methods have been investigated for fabricating FPIs with polymer spacers. The first involves forming an FPI by depositing a reflective aluminium coating on either side of a discrete polyethylene terepthalate film that then is bonded to a glass or polymer substrate (typically a centimeter or two thick) using an optically transparent adhesive [6]. More recently, spin coating [8] and gas phase vacuum deposition techniques, such as the Parylene process [4], [12], have been used to directly deposit the polymer spacer on to a substrate precoated with metallic or dielectric layers that form the first FPI mirror. The second mirror then is deposited on to the polymer spacer. The use of the Parylene process, in particular, to form the polymer layer, holds a number of advantages. It provides a high degree of thickness uniformity and excellent surface finish, both of which are required to form a high-quality FPI and allows the sensors to be inexpensively batch fabricated with a high degree of repeatability. For these reasons, sensors fabricated in this way provide an example of practical importance to study and are considered in this paper.

As indicated in the previous section, the transduction mechanism may be considered to consist of two parts: the sensitivity of the light-intensity modulation to a change in 
optical phase (the intensity-phase transfer function mentioned above), and the sensitivity of the optical phase to the incident acoustic wave. The former can be described using the well established descriptions of multiple beam interferometry used to model transfer functions of FPIs [15]. The latter is the subject of this paper, as its dependence on the direction and frequency of an incoming plane acoustic wave determines the directionality and frequency response of the sensor.

\section{MODEL OF DiRECTIVITY AND FREQUENCY RESPONSE}

As described above, the FP sensor can detect ultrasound because an acoustic wave traveling through the sensor changes the thickness of the polymer layer. This results in a change in the phase between the light reflected from the surfaces of the polymer layer, which alters the reflected light intensity. It is this modulated light intensity that is measured and from which the acoustic pressure is inferred. The frequency and angle of incidence of the incident wave will affect the amount by which the thickness of the polymer layer changes. Therefore, the sensor response exhibits some directionality. This section describes a model to calculate this frequency-dependent directional response.

\section{A. Optical Phase Change}

In the absence of an acoustic wave, the difference in phase between the light reflected from the two sides of the interferometer is $\phi=4 \pi n d / \lambda_{0}$ radians, where $n$ is the refractive index, $d$ is the thickness of the polymer film, and $\lambda_{0}$ is the in vacuo wavelength of the interrogating light beam. A change in this phase could be due to a physical change in the thickness of the film or a change in its refractive index. As the passage of an acoustic wave through the sensor could, in principle, produce either effect, there are two possible mechanisms by which the sound wave could cause a modulation of the intensity of the reflected beam. In this work, the second mechanism - the refractive index change - was assumed to be negligible; the reasons for this are discussed in the Appendix. The optical phase change, therefore, is modeled as due only to the first mechanism: a change in the physical thickness of the sensor as the acoustic wave propagates through it. To a linear approximation, the change in phase, $\Delta \phi$, may be written as:

$$
\Delta \phi=\frac{\partial \phi}{\partial d} \Delta d=\left(\frac{4 \pi n}{\lambda_{0}}\right) \frac{\partial d}{\partial p} \Delta p
$$

where $\Delta d$ is a change in the thickness due to a change in the external acoustic pressure $\Delta p . \partial \phi / \partial d=4 \pi n / \lambda_{0}$ is the sensitivity of the phase to a change in the thickness, and $\partial d / \partial p$ is the sensitivity of the thickness to a change in pressure. As the change in thickness, $\Delta d$, is the difference in the changes in the vertical particle displacements, $u_{z}$, on the two sides, $z=0$ and $z=d$, of the FP layer, the sensitivity of the thickness to a change in pressure may be written:

$$
\frac{\partial d}{\partial p}=\frac{u_{z}(d)-u_{z}(0)-d}{\Delta p} .
$$

In other words, for a unit amplitude incident wave, the sensitivity of interest, $\partial d / \partial p$, is just the difference in the particle displacements at the two sides of the polymer film minus its unperturbed thickness. In Section III-B, a model of elastic waves in solids is used to calculate this sensitivity as a wave travels through the sensor. $\partial d / \partial p$, averaged over the illuminated area of the sensor, weighted by the laser beam profile $S(x)$, gives the frequency-dependent directional response of the sensor:

$$
D(f, \theta)=\frac{\int_{-\infty}^{\infty}(\partial d / \partial p) S(x) d x}{\int_{-\infty}^{\infty} S(x) d x} .
$$

\section{B. Elastic Wave Model}

The sensor was treated as a linear, elastic, layered medium characterized by two elastic constants per layer, e.g., Lamé's parameters $\lambda$ and $\mu$, or Young's modulus $E$ and Poisson's ratio $\sigma$. It was assumed to be infinitely wide, with physical properties unvarying in the horizontal direction, and with a semi-infinitely thick substrate. By taking advantage of the planar geometry of the sensor in this way, an analytical model can be formulated that provides considerably greater computational efficiency than numerical models based on less specific approaches, such as finite difference or finite-element schemes [16]. It also was assumed that the optically reflecting layers forming the FP cavity were much thinner than an acoustic wavelength and, therefore, acoustically negligible. This is a good assumption for the type of sensors and frequency ranges used in the measurements, Section IV, and allows for a simpler mathematical description. For higher frequencies in which this assumption becomes invalid, the model may be straightforwardly extended to include these extra layers.

The equation for the time-varying vector particle displacement, $\mathbf{u}=\left(u_{x}, u_{y}, u_{z}\right)$, in an isotropic, elastic, material is well known [17]:

$$
\rho \frac{\partial^{2} \mathbf{u}}{\partial t^{2}}=(\lambda+2 \mu) \nabla(\nabla \cdot \mathbf{u})-\mu \nabla \times(\nabla \times \mathbf{u}) .
$$

The task here is to calculate the vertical particle displacement on the boundaries of the polymer (interferometer) layer, $u_{z}(0)$ and $u_{z}(d)$, by solving (4) within each layer subject to appropriate matching conditions on the boundaries at which the layers meet. A standard method of achieving this is described in [17]-[19].

The displacement vector may be written as the sum $\mathbf{u}=\nabla \phi+\nabla \times \boldsymbol{\psi}$, where $\phi$ and $\boldsymbol{\psi}$ are scalar and vector potentials, respectively. In an isotropic medium, (4) then splits into two wave equations: one for the scalar potential, 


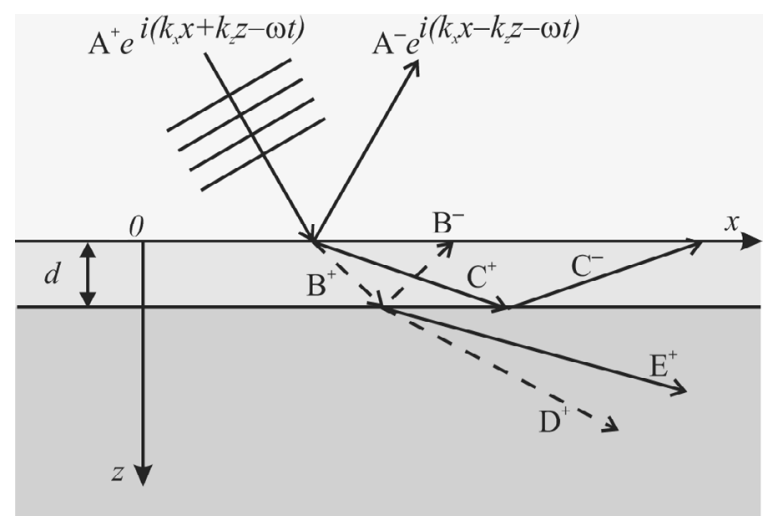

Fig. 2. The coordinate system and notation for the wave approach to characterizing the acoustic response of the sensor. The FP layer has thickness $d$ in the absence of an acoustic wave. The solid lines are compressional (P) waves and the dotted lines shear waves. Assuming an incident wave of known amplitude, there are seven unknown wave amplitudes $\left(A^{-}, B^{ \pm}, C^{ \pm}, D^{+}, E^{+}\right)$and so seven boundary conditions must be specified.

which describes longitudinal waves traveling with speed $c_{l}=\sqrt{(\lambda+2 \mu) / \rho}$, and one for the vector potential, which describes shear waves with speed $c_{s}=\sqrt{\mu / \rho}$ :

$$
\begin{aligned}
\frac{\partial^{2} \phi}{\partial t^{2}}-c_{l}^{2} \nabla^{2} \phi & =0, \\
\frac{\partial^{2} \boldsymbol{\psi}}{\partial t^{2}}-c_{t}^{2} \nabla^{2} \boldsymbol{\psi} & =0 .
\end{aligned}
$$

The shear wave propagates as a transverse wave, and $\psi$ points in a direction perpendicular to both the displacement $\nabla \times \boldsymbol{\psi}$ and the direction of travel. Any shear wave may be considered as a combination of horizontally and vertically polarized shear waves in which the particle motion is in the horizontal or vertical plane, respectively. The two-dimensional (2-D) model described below, and shown in Fig. 2, does not include horizontally polarized shear waves; therefore bulk shear horizontal (SH) and Love waves are excluded. This is not a limitation, however, as such waves by definition do not cause displacements in the $z$ direction, which are the only movements of interest for this application. With this simplification, the vector potential can be written, without loss of generalization, as $\boldsymbol{\psi}=(0, \psi, 0)$, where $\psi$ is a scalar. In this case, the displacement vector becomes:

$$
\mathbf{u}=\left(\frac{\partial \phi}{\partial x}-\frac{\partial \psi}{\partial z}, \frac{\partial \phi}{\partial y}, \frac{\partial \phi}{\partial z}+\frac{\partial \psi}{\partial x}\right)
$$

Single-frequency, plane wave, solutions to the wave equations (5) and (6) will take the forms:

$$
\begin{aligned}
& \phi \propto e^{i(\mathbf{k} \cdot \mathbf{x}-\omega t)}, \\
& \psi \propto e^{i\left(\mathbf{k}_{t} \cdot \mathbf{x}-\omega t\right)},
\end{aligned}
$$

where $\omega$ is the temporal frequency, $t$ is the time, $\mathbf{x}=$ $(x, y, z)$ a position vector, and $\mathbf{k}=\left(k_{x}, k_{y}, k_{z}\right)$ and $\mathbf{k}_{t}=$ $\left(k_{t x}, k_{t y}, k_{t z}\right)$ are wavevectors for the compressional (longitudinal) and shear waves, respectively. Here we study a
2-D model (Fig. 2) because, for a plane wave incident at any angle $\theta$ on another plane, the coordinate axes always can be aligned with the wavefront to reduce the problem from three to two dimensions. Here the axes have been chosen such that $k_{y}=k_{t y}=u_{y}=0$.

As a plane pressure wave propagates through the sensor, part of it will be reflected and part transmitted at each boundary. No reflections occur other than at the boundaries between the layers because the sensor's physical properties were assumed to be constant in the horizontal direction. In the steady state, all the (multiply reflected) upward-traveling compressional waves within the polymer layer can be summed and treated as one wave with a complex amplitude, $C^{-}$. Similarly, all the downward traveling waves can be lumped together with the complex amplitude, $C^{+}$. In this way, the field in the polymer layer can be described by four wave amplitudes, $B^{ \pm}$and $C^{ \pm}$, corresponding to downward/upward traveling shear and compressional waves, respectively. A similar approach and notation is used to describe the wave amplitudes in the surrounding media too. Surface waves, such as leaky Rayleigh waves, are implicitly included in this model as they are combinations of shear and compressional motions. The medium above the sensor is fluid, chosen here to be water, so it does not support shear waves. In the polymer layer and the substrate, however, both shear (dotted) and compressional (solid) waves are excited (see Fig. 2). If, with no loss of generality, the incident pressure wave is assigned unit amplitude, there are seven unknown wave amplitudes, denoted in Fig. 2 by $A^{-}, B^{ \pm}, C^{ \pm}, D^{+}$, and $E^{+}(+$indicates waves traveling in the positive $z$ direction. $A, C$, and $E$ are amplitudes of compressional waves and $B$ and $D$ of shear waves). If the displacements in the three layers of the model are denoted with subscripts 1, 2, and 3 for water, polymer layer, and substrate, respectively, the displacement potentials may be written, for this 2-D case as:

$$
\begin{aligned}
& \phi_{1}=\Psi\left(A^{+} e^{i k_{z 1} z}+A^{-} e^{-i k_{z 1} z}\right), \\
& \psi_{2}=\Psi\left(B^{+} e^{i k_{t z 2} z}+B^{-} e^{-i k_{t z 2} z}\right), \\
& \phi_{2}=\Psi\left(C^{+} e^{i k_{z 2} z}+C^{-} e^{-i k_{z 2} z}\right), \\
& \psi_{3}=\Psi D^{+} e^{i k_{t z 3} z}, \\
& \phi_{3}=\Psi E^{+} e^{i k_{z 3} z},
\end{aligned}
$$

where the common factor $\Psi=\exp \left(i\left(k_{x} x-\omega t\right)\right)$ appears because the wavenumbers in the $x$ direction, $k_{x}$, always must be the same as that of the incident wave (Snell's law). Eq. (10)-(14) are for displacement potentials, so the known amplitude $A^{+}=P_{0} /\left(\rho_{1} \omega^{2}\right)$, where $P_{0}$ is the incident pressure amplitude. Here, we set $P_{0}=1$.

To solve for the seven unknown amplitudes, seven conditions of continuity at the boundaries are required. It is assumed that the solid polymer layer and solid substrate are in welded contact, but that the fluid can slip over the solid. This results in the following seven requirements:

- the normal particle displacement is continuous across both boundaries (1 and 2), 
- the tangential particle displacement is continuous across the polymer layer/substrate boundary (3),

- the normal stress is continuous across both boundaries (4 and 5),

- the shear stress is continuous across both boundaries (6 and 7).

Conditions 4-7 are written in terms of stress. The displacement-strain and strain-stress relationships are well-known and allow the seven conditions to be rewritten as:

$$
\begin{aligned}
& u_{z 1}(0)=u_{z 2}(0) \\
& u_{z 2}(d)=u_{z 3}(d) \\
& u_{x 2}(d)=u_{x 3}(d) \\
& \lambda_{1}\left(\left.\frac{\partial u_{x 1}}{\partial x}\right|_{0}+\left.\frac{\partial u_{z 1}}{\partial z}\right|_{0}\right)=\left.\lambda_{2} \frac{\partial u_{x 2}}{\partial x}\right|_{0}+\left.\nu_{2} \frac{\partial u_{z 2}}{\partial z}\right|_{0} \\
&\left.\lambda_{2} \frac{\partial u_{x 2}}{\partial x}\right|_{d}+\left.\nu_{2} \frac{\partial u_{z 2}}{\partial z}\right|_{d}=\left.\lambda_{3} \frac{\partial u_{x 3}}{\partial x}\right|_{d}+\left.\nu_{3} \frac{\partial u_{z 3}}{\partial z}\right|_{d} \\
& \mu_{2}\left(\left.\frac{\partial u_{x 2}}{\partial z}\right|_{0}+\left.\frac{\partial u_{z 2}}{\partial x}\right|_{0}=0,\right. \\
& \mu_{2}\left(\left.\frac{\partial u_{x 2}}{\partial z}\right|_{d}+\left.\frac{\partial u_{z 2}}{\partial x}\right|_{d}\right)=\mu_{3}\left(\left.\frac{\partial u_{x 3}}{\partial z}\right|_{d}+\left.\frac{\partial u_{z 3}}{\partial x}\right|_{d}\right)
\end{aligned}
$$

where $\nu=2 \mu+\lambda$. Substituting expressions for the potentials obtained from (10)-(14) and (7), into these matching conditions results in seven equations in the seven unknown amplitudes $A-E$. These can be written in matrix form as (22) (see next page), where $e_{1}=\exp \left(i k_{t z 2} d\right)$, $e_{2}=\exp \left(i k_{z 2} d\right), e_{3}=\exp \left(i k_{t z 3} d\right)$, and $e_{4}=\exp \left(i k_{z 3} d\right)$. The following shorthand also was used: $\bar{\lambda}_{2}=\lambda_{2}-\nu_{2}$, $\bar{\lambda}_{3}=\left(\lambda_{3}-\nu_{3}\right), \gamma_{2}=\lambda_{2} k_{x}^{2}+\nu_{2} k_{z 2}^{2}$, and $\gamma_{3}=\lambda_{3} k_{x}^{2}+\nu_{3} k_{z 3}^{2}$. This matrix equation may be solved efficiently using standard matrix techniques [18], [19] to yield the unknown wave amplitudes $A^{-}, \ldots, E^{+}$. Using (7), (11), and (12), the vertical component of the displacement vector within the polymer layer may be written:

$$
\begin{aligned}
u_{z 2}(x, z)= & \frac{\partial \phi_{2}}{\partial z}+\frac{\partial \psi_{2}}{\partial x} \\
= & i k_{z 2} \Psi(x)\left(C^{+} e^{i k_{z 2} z}-C^{-} e^{-i k_{z 2} z}\right) \\
& +i k_{x} \Psi(x)\left(B^{+} e^{i k_{t z 2} z}+B^{-} e^{-i k_{t z 2} z}\right) .
\end{aligned}
$$

Once the system of equations has been solved for the seven unknown amplitudes using (22) the difference in vertical particle displacements $u_{z 2}(0)-u_{z 2}(d)$ can be ascertained, and the required sensitivity $\partial d / \partial p$ can be calculated from (2) as a function of frequency $\omega$ and incidence angle $\theta=$ $\sin ^{-1}\left(k_{x} / k\right)$ where $k=\omega / c$. The final task in calculating the sensor response is to average over the area of the spot illuminated by the interrogating laser, taking into account the laser beam profile.

\section{Spatial Averaging Over the Beam Profile}

The interrogating laser beam has a finite spot radius, so rather than measuring at one point on the sensor, it measures the spatial average over the spot. This averaging will affect the directional response of the sensor, i.e., the response to a wave incident at an oblique angle $\theta \neq 0$.

In $(23)$ the factor $\Psi(x)$ contains all the $x$-dependence, so the integral in the numerator of (3) becomes the integral of $\Psi(x) S(x)$ over $x$, where $S$ describes the spot size and profile. $S$ could describe any beam profile, such as a Gaussian, but here a top-hat beam of radius $a$ is used:

$$
\begin{aligned}
S(x) & =1 & & \text { for }-a \leq x \leq a \\
& =0 & & \text { elsewhere. }
\end{aligned}
$$

Averaging over $x$ gives the normalized directivity factor $\bar{\Psi}$ as:

$$
\bar{\Psi}=\frac{\int_{-\infty}^{\infty} \Psi(x) S(x) d x}{\int_{-\infty}^{\infty} S(x) d x}
$$

For the top-hat case in 2-D this becomes:

$$
\bar{\Psi}=\frac{1}{2 a} \int_{-a}^{a} e^{i k_{x} x} d x=\frac{\sin \left(k_{x} a\right)}{k_{x} a}
$$

where the time-dependent phase factor $\exp (-i \omega t)$ has been omitted for convenience.

In 3-D Cartesian coordinates $(x, y, z)$, the profile $S$, as given in (25), represents a line source of width $2 a$, infinitely long in the $y$ direction. In order to model a circular spot, rather than a line source, the beam profile must be described as a function of a radial coordinate, $r=\sqrt{x^{2}+y^{2}}$. If $r$ is chosen to be zero at the center of the beam, then the beam profile for the top-hat circular beam is the same as (25), except with $x$ replaced by $r=\sqrt{x^{2}+y^{2}}$. By transforming from the Cartesian coordinates $(x, y)$ to the cylindrical polar coordinates $(r, \zeta)$, the corresponding directivity factor becomes:

$$
\begin{aligned}
\bar{\Psi} & =\frac{1}{\pi a^{2}} \int_{0}^{a} \int_{0}^{2 \pi} e^{i k_{x} r \cos \zeta} r d \zeta d r \\
& =\frac{2}{a^{2}} \int_{0}^{a} J_{0}\left(k_{x} r\right) r d r=\frac{2 J_{1}\left(k_{x} a\right)}{k_{x} a} .
\end{aligned}
$$

So, using (2) and (3), the overall directivity $D(f, \theta)$ now can be written:

$$
\begin{aligned}
& D=i k_{z 2} \bar{\Psi}\left(C^{+}\left(e^{i k_{z 2} d}-1\right)-C^{-}\left(e^{-i k_{z 2} d}-1\right)\right) \\
& +i k_{x} \bar{\Psi}\left(B^{+}\left(e^{i k_{t z 2} d}-1\right)+B^{-}\left(e^{-i k_{t z 2} d}-1\right)\right)-d .
\end{aligned}
$$

Eq. (30) can be used to calculate the complete, frequencydependent, complex directional response, i.e., both magnitude and phase.

\section{Directivity Measurements}

As a first test of the model described in Section III, it was compared to a previous, experimentally validated 


$\left[\begin{array}{c}k_{z 1} A^{+} \\ 0 \\ 0 \\ \lambda_{1} k_{1}^{2} A^{+} \\ 0 \\ 0 \\ 0\end{array}\right]=\left[\begin{array}{ccccccc}k_{z 1} & k_{x} & k_{x} & k_{z 2} & -k_{z 2} & 0 & 0 \\ 0 & k_{x} e_{1} & k_{x} / e_{1} & k_{z 2} e_{2} & -k_{z 2} / e_{2} & -k_{x} e_{3} & -k_{z 3} e_{4} \\ 0 & -k_{t z 2} e_{1} & k_{t z 2} / e_{1} & k_{x} e_{2} & k_{x} / e_{2} & k_{t z 3} e_{3} & -k_{x} e_{4} \\ -\lambda_{1} k_{1}^{2} & -\bar{\lambda}_{2} k_{x} k_{t z 2} & \bar{\lambda}_{2} k_{x} k_{t z 2} & \gamma_{2} & \gamma_{2} & 0 & 0 \\ 0 & \bar{\lambda}_{2} k_{x} k_{t z 2} e_{1} & -\bar{\lambda}_{2} k_{x} k_{t z 2} / e_{1} & -\gamma_{2} e_{2} & -\gamma_{2} / e_{2} & -\bar{\lambda}_{3} k_{x} k_{t z 3} e_{3} & \gamma_{3} e_{4} \\ 0 & \mu_{2}\left(k_{t z 2}^{2}-k_{x}^{2}\right) & \mu_{2}\left(k_{t z 2}^{2}-k_{x}^{2}\right) & -2 \mu_{2} k_{x} k_{z 2} & 2 \mu_{2} k_{x} k_{z 2} & 0 & 0 \\ 0 & \mu_{2}\left(k_{t z 2}^{2}-k_{x}^{2}\right) e_{1} & \mu_{2}\left(k_{t z 2}^{2}-k_{x}^{2}\right) / e_{1} & -2 \mu_{2} k_{x} k_{z 2} e_{2} & 2 \mu_{2} k_{x} k_{z 2} / e_{2}-\mu_{3}\left(k_{t z 3}^{2}-k_{x}^{2}\right) e_{3} 2 \mu_{3} k_{z 3} k_{x} e_{4}\end{array}\right]\left[\begin{array}{c}A^{-} \\ B^{+} \\ B^{-} \\ C^{+} \\ C^{-} \\ D^{+} \\ E^{+}\end{array}\right]$

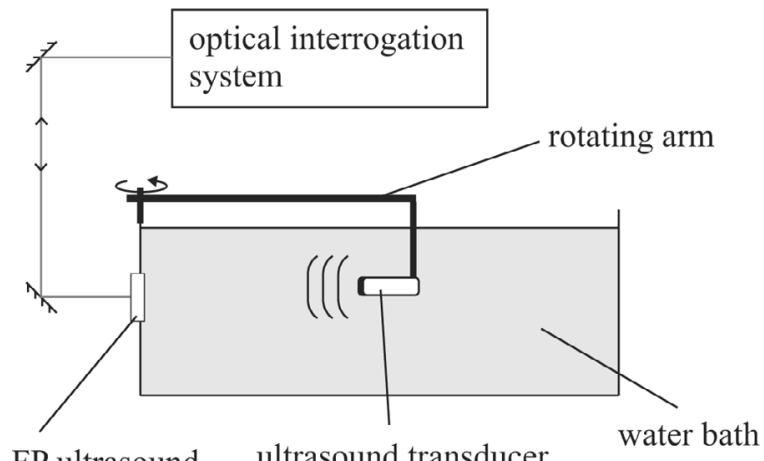

FP ultrasound ultrasound transducer sensor

Fig. 3. Experimental arrangement for the plane-wave directivity measurements.

model [6], which describes the sensor's normal-incidence frequency response. This other model has been shown to agree with normal-incidence, plane-wave, pressure measurements for a variety of different substrates and polymer layers. Both models give the same normal-incidence response; the model described here, however, can also account for waves with oblique angles of incidence. An experiment with which to compare the model output for obliquely incident waves was therefore devised.

Fig. 3 shows the experimental arrangement used to measure the directional response of the detector, i.e., the response to plane waves incident at oblique angles. A Panametrics V312-SU ultrasound transducer (6-mm diameter, $10 \mathrm{MHz}$ center frequency, $-6 \mathrm{~dB}$ points at 6.3 and $12.9 \mathrm{MHz}$ ) driven with a Panametrics $5052 \mathrm{PR}$ pulserreceiver was mounted on an arm capable of being rotated such that the sound beam from the transducer always was incident on the same point on the sensor, from the same distance away. To ensure that the wavefronts arriving at the sensor were plane, the transducer was placed in the far field, $140 \mathrm{~mm}$ from the sensor face. This is in the far field for frequencies up to about $20 \mathrm{MHz}$. The maximum output of the transducer was $130 \mathrm{kPa}$, well within the linear range of the sensor [12]. The sensor used in these measurements, described in detail in [12], consisted of a 3.8-mm thick substrate of borosilicate glass $\left(c_{l} \approx 5640 \mathrm{~m} / \mathrm{s}, c_{s} \approx 3280 \mathrm{~m} / \mathrm{s}\right.$, $\rho=2240 \mathrm{~kg} / \mathrm{m}^{3}$ ) and a $40-\mu \mathrm{m}$ thick Parylene C (Specialty Coatings Systems, Indianapolis, IN) polymer layer $\left(c_{l} \approx 2200 \mathrm{~m} / \mathrm{s}, c_{s} \approx 1100 \mathrm{~m} / \mathrm{s}, \rho=1180 \mathrm{~kg} / \mathrm{m}^{3}\right)$. The reflective coatings were aluminium in this case and with a thickness of $\approx 50 \mathrm{~nm}$ were considered acoustically negligible. The lateral dimensions of the sensor were $5 \times 3 \mathrm{~cm}$. The laser beam used to interrogate the sensor was a $70 \mathrm{~mW}$, $850 \mathrm{~nm}$, distributed Bragg reflector laser diode. Its output was collimated and expanded to provide a large area elliptical beam of dimensions $16 \times 12 \mathrm{~mm}$. The bias point to ensure a linear response was chosen by angle tuning the incident beam [12], [20]. The light reflected from the sensor was directed through an aperture, $400-\mu \mathrm{m}$ radius, to give a top-hat profile, to a photodiode. The high-pass filtered $(>300 \mathrm{kHz}$ ) output of which was recorded using a $500 \mathrm{MHz}$ digitizing oscilloscope. The arm was manually rotated in steps of $1^{\circ}$, and a measurement of the acoustic signal from the transducer was made at each angle (averaged over 100 pulses). These measurements were then Fourier transformed to give the frequency-angle sensor response.

Fig. 4 shows a measurement of the frequency-angle response for angles from $0^{\circ}$ (normal incidence) to $80^{\circ}$ for a frequency range from 1 to $15 \mathrm{MHz}$. These measurements were normalized to the frequency response at normal incidence predicted by the model. The prediction of the model, using the estimated material parameters stated above, is shown in Fig. 5. The minima that form the series of concave curves are due to spatial averaging over the illuminated spot. This occurs when the horizontal component of the wavelength is an integer multiple of the spot diameter, i.e., at the zeros of $\bar{\Psi}(29)$, which is approximately $k_{x} a=\pi(n+1 / 4)$ for $n=1,2, \ldots$ The two vertical features, labeled A and B, are caused by critical angle effects. At low frequency, these minima occur close to the critical angles for transmission of compressional $\left(15^{\circ}\right)$ and shear waves $\left(27^{\circ}\right)$ from water to glass, a consequence of the fact that, at these low frequencies, the polymer layer is much thinner than a wavelength and thus loses its ability to influence the waves' behavior. At these critical angles, the wavefronts are traveling horizontally in the glass and, hence, almost horizontally in the polymer. Therefore, there is little displacement in the vertical direction and the sensor response falls to a minimum. At higher frequencies, more complex interference effects affect the response, as the polymer layer becomes a significant proportion of a wavelength.

The comparison between the measurement and the model can be seen more clearly in the horizontal profiles through Figs. 4 and 5 for frequencies from 1 to $13 \mathrm{MHz}$, 


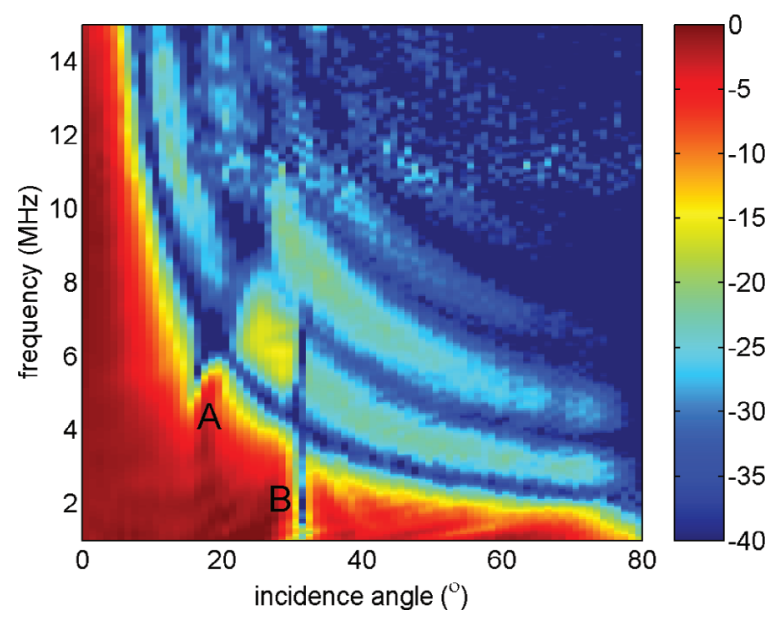

Fig. 4. The measured, plane-wave, frequency-angle response in decibels, for a frequency range of $1-15 \mathrm{MHz}$ and incidence angles of $0-80^{\circ}$, for a glass-backed, Parylene film FP ultrasound sensor with aluminium reflective coatings and a $40-\mu \mathrm{m}$ Parylene $\mathrm{C}$ layer. The response has been normalized to show the same normal incidence response as the model, shown in Fig. 5.

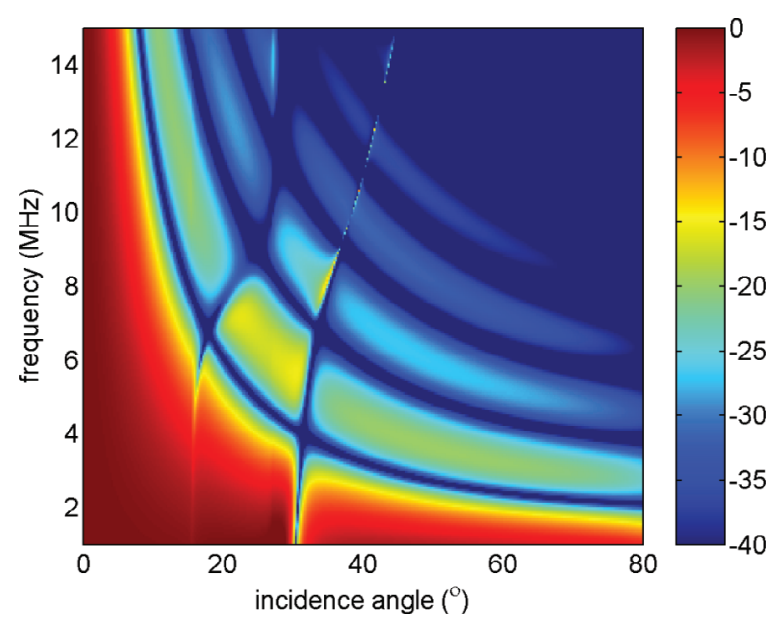

Fig. 5. The plane-wave, frequency-angle response of a glass-backed sensor predicted by the model of Section III, and corresponding to the measurements in Fig. 4. The parameters used were: $40-\mu \mathrm{m}$ Parylene C layer $\left(c_{l}=2200 \mathrm{~m} / \mathrm{s}, c_{s}=1100 \mathrm{~m} / \mathrm{s}, \rho=1180 \mathrm{~kg} / \mathrm{m}^{3}\right)$, glass substrate $\left(c_{l}=5640 \mathrm{~m} / \mathrm{s}, c_{s}=3280 \mathrm{~m} / \mathrm{s}, \rho=2240 \mathrm{~kg} / \mathrm{m}^{3}\right)$, and $400-\mu \mathrm{m}$ radius top-hat interrogation beam.

shown in Fig. 6. From these directivity plots and the full, frequency-angle plots, it is clear that there is good qualitative agreement between the model and the measurements overall and good quantitative agreement at lower frequencies and smaller incidence angles. However, there are discrepancies in the amplitude if not the shape of the response at higher angles of incidence and higher frequencies, largely due to signal-to-noise limitations. Three factors contribute to the low signal-to-noise ratio (SNR): the reduced output of the transducer at higher frequencies (at $15 \mathrm{MHz}$ it is $10 \mathrm{~dB}$ below its maximum output at $10 \mathrm{MHz}$ ), the increased attenuation at higher frequencies and the large $(140 \mathrm{~mm})$ transducer-sensor distance leads to at least a further $7 \mathrm{~dB}$ of attenuation, and the sensor itself is less
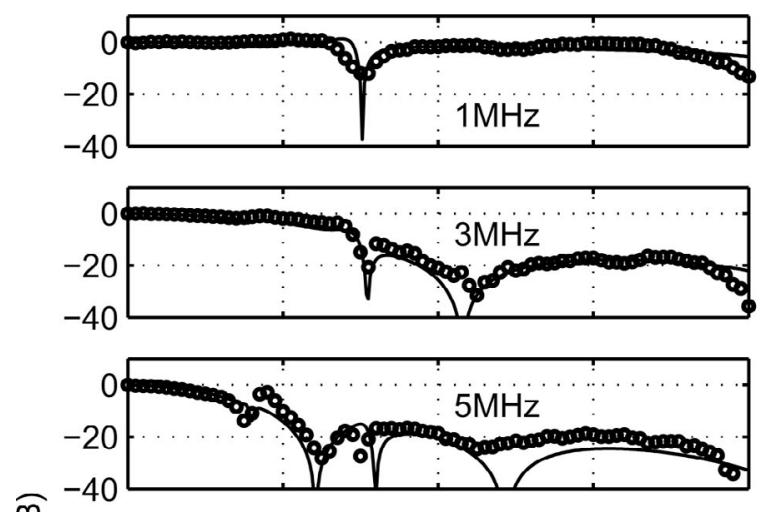

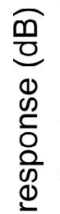
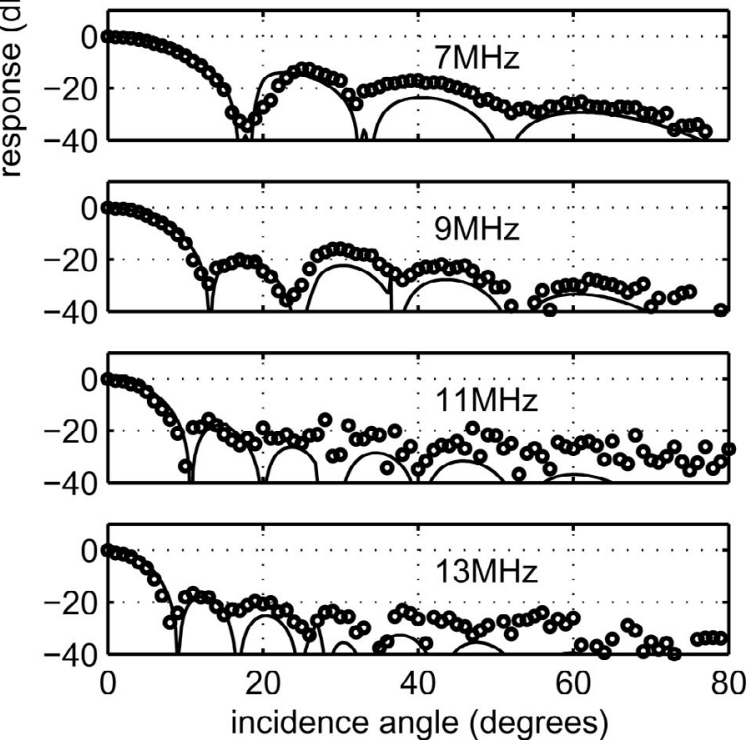

Fig. 6. Directivity plots at frequencies of $1-13 \mathrm{MHz}$ obtained by taking horizontal profiles through Figs. 4 and 5 and normalizing to normal incidence. '-' model, 'o' measurements.

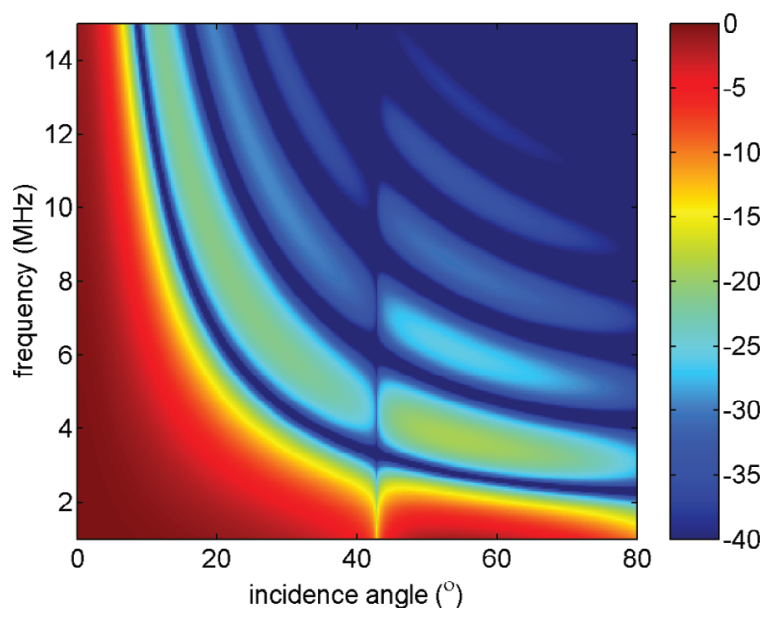

Fig. 7. The plane-wave, frequency-angle response of a polycarbonatebacked sensor predicted by the model of Section III. The parameters used were: $40-\mu \mathrm{m}$ Parylene C layer $\left(c_{l}=2200 \mathrm{~m} / \mathrm{s}, c_{s}=1100 \mathrm{~m} / \mathrm{s}\right.$, $\left.\rho=1180 \mathrm{~kg} / \mathrm{m}^{3}\right)$, polycarbonate substrate $\left(c_{l}=2180 \mathrm{~m} / \mathrm{s}, c_{s}=\right.$ $\left.960 \mathrm{~m} / \mathrm{s}, \rho=1180 \mathrm{~kg} / \mathrm{m}^{3}\right), 400-\mu \mathrm{m}$ radius top-hat interrogation beam. 
sensitive at large angles of incidence and high frequencies. The large transducer-sensor separation was a necessary requirement to ensure that the sensor was in the transducer's far field so that the wavefronts were essentially plane when they reached it. As well as the noise introduced by the low SNR, there was some experimental error due to the difficulty of aligning the experimental system to ensure that the illuminated, sensitive point on the detector remained at the same point in the field of the ultrasound transducer for every measurement angle.

\section{Applichtions}

\section{A. Sensor Design}

Perhaps the most direct application of the model is its use as a predictive tool to improve the sensor design itself through careful investigation of the effects of the material and geometric parameters. For instance, by replacing the glass substrate with polycarbonate $\left(c_{l}=2180 \mathrm{~m} / \mathrm{s}\right.$, $c_{s}=960 \mathrm{~m} / \mathrm{s}, \rho=1180 \mathrm{~kg} / \mathrm{m}^{3}$ ), which has a lower shear speed than the sound speed in the water, the vertical feature, B, can be eliminated, Fig. 7. The minimum in Fig. 7 at $42^{\circ}$ corresponds to the compressional wave critical angle, feature A in Fig. 4, although clearly shifted from $15^{\circ}$ because of the slower wave speed. At the critical angle the compressional wave is traveling perpendicular to the sensor inside the polymer layer and so causes no motion in the vertical direction. The minimum remains at a constant angle as the frequency increases because the acoustic properties of the polycarbonate and Parylene are similar (so there is no significant acoustic boundary between them); therefore, even when the polymer layer is a significant proportion of a wavelength, interference effects do not dominate ( $d$ is no longer a characteristic length scale for the problem).

\section{B. Effective Element Radius}

It is often assumed that the size of the acoustically sensitive measuring "element" is given by the size of the region illuminated with the interrogation light beam. In fact, a signal arriving at the sensor outside the illuminated area may propagate as a surface wave to the illuminated region and be detected. This phenomenon has also been noted in piezoelectric PVDF detectors such as membrane hydrophones, in which Lamb waves propagate to the sensitive region from outside it. These guided surface waves and other modes cause the directivity of the sensor to deviate from that of a purely spatially-averaging pressure detector. The "effective" element radius is a useful but approximate measure of a sensor's directional characteristics [21], [22], obtained by comparing the directivity of the real sensor to the directivity of a rigid, circular, pressure detector of radius $a_{\text {eff }}$, whose directivity is due purely to spatial averaging and is given by:

$$
D_{\mathrm{eff}}=\frac{2 J_{1}\left(k_{x} a_{\mathrm{eff}}\right)}{k_{x} a_{\mathrm{eff}}}
$$
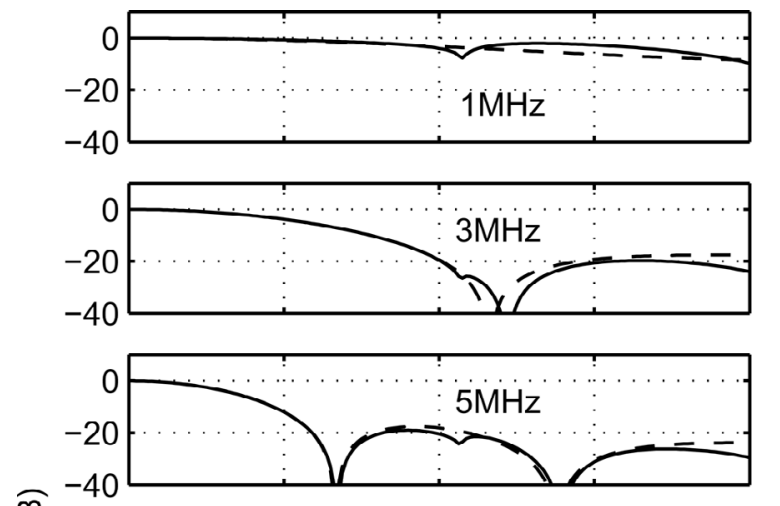

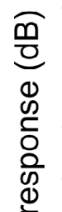
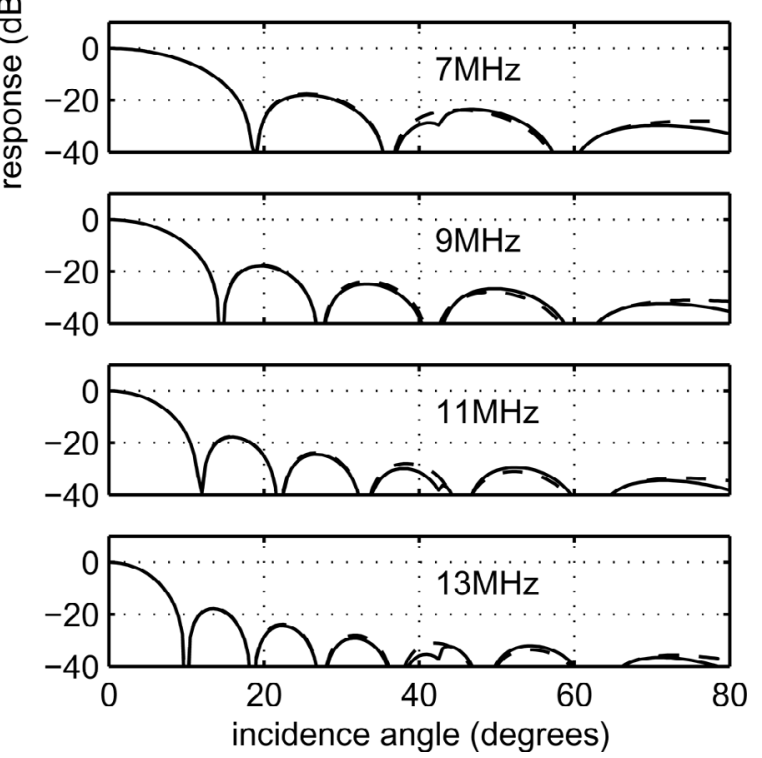

Fig. 8. Directivity plots at frequencies of $1-13 \mathrm{MHz}$ obtained by taking horizontal profiles through Fig. 7 and normalizing to normal incidence. Also shown is the directivity function $J_{1}\left(k_{x} a_{\mathrm{eff}}\right) /\left(k_{x} a_{\mathrm{eff}}\right)$, for an ideal, rigid, circular, pressure detector of radius $a=400 \mu \mathrm{m}$ (dashed line) indicating that, in this case, the effective element radius equals the illuminated spot radius.

The value of $a_{\text {eff }}$ for which $D_{\text {eff }}\left(a_{\text {eff }}\right)$ most closely matches the measured directivity is called the effective element radius, irrespective of the radius of the illuminated spot. The effective and optically defined element radii differ when the sensor does not behave like a rigid, circular, pressure detector. In this section the directivity model is used to answer two, related, questions:

- Under what conditions is the effective element radius the same as the illumination spot radius?

- Is there a minimum, attainable, effective element radius for a given sensor thickness $d$ ? Is there a limit beyond which a smaller illumination spot radius makes no difference to the directivity pattern?

In Fig. 8 the solid lines show the directivity of a 40$\mu \mathrm{m}$ thick, polycarbonate-backed sensor with a circular illuminated spot of $400-\mu \mathrm{m}$ radius (i.e., horizontal profiles through Fig. 7), and the dashed lines are the function $D_{\text {eff }}$, also with radius $a_{\text {eff }}=400 \mu \mathrm{m}$. The good agreement between the two directivity functions suggests that, in this case, with an illuminated spot radius much larger than the 


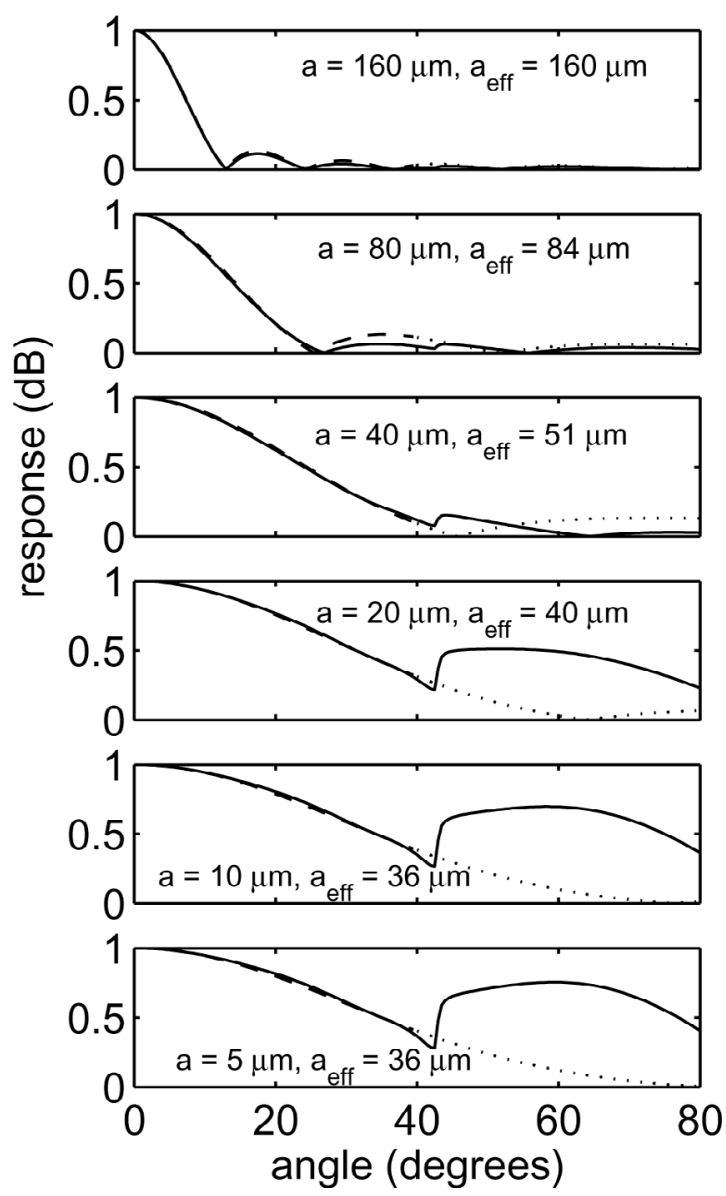

Fig. 9. Directivity plots from the model (solid line) of a $40-\mu \mathrm{m}$ thick, polycarbonate-backed sensor at a frequency of $25 \mathrm{MHz}$ for illumination spot radii of $160,80,40,20,10$, and $5 \mu \mathrm{m}$ shown on a linear scale. Also shown (dashed-dotted line) is the function $J_{1}\left(k_{x} a_{\text {eff }}\right) /\left(k_{x} a_{\text {eff }}\right)$ at which the radius $a_{\text {eff }}$ has been found through a best-fit over angles from 0 to $40^{\circ}$ (the dashed part of the line).

sensor layer thickness, the effective element radius corresponds closely to the illumination spot radius, at least for frequencies above $3 \mathrm{MHz}$, confirming our intuitive expectation.

Fig. 9 shows an example in which the approximation $a_{\text {eff }} \approx a$ begins to break down as the illuminating spot radius is reduced. The solid lines show the directivity, on a linear scale, at $25 \mathrm{MHz}$ for the $40-\mu \mathrm{m}$ sensor for decreasing illumination spot radii of $160,80,40,20,10$, and $5 \mu \mathrm{m}$ (from 4 to $1 / 8$ times the FP layer thickness). Eq. (31) was fitted to these directivity patterns in a least-squares sense for angles from 0 to $40^{\circ}$, as at larger angles the rigid disc approximation differs significantly from the sensor model, and the comparison would have been meaningless. Similarly, approximate but useful small-angle comparisons have been made in the past for hydrophone directivities, by comparing the widths of the first lobe (beamwidths) and ignoring higher-angle effects [21], [22]. In Fig. 9 we see that, for large $a$, the spatial-averaging term, $\bar{\Psi}$ from (29), dominates the behavior, and we see good agreement between $a_{\text {eff }}$ and $a$. As $a$ is reduced in size, $\bar{\Psi}$ approaches unity and interference effects within the sensor affect the

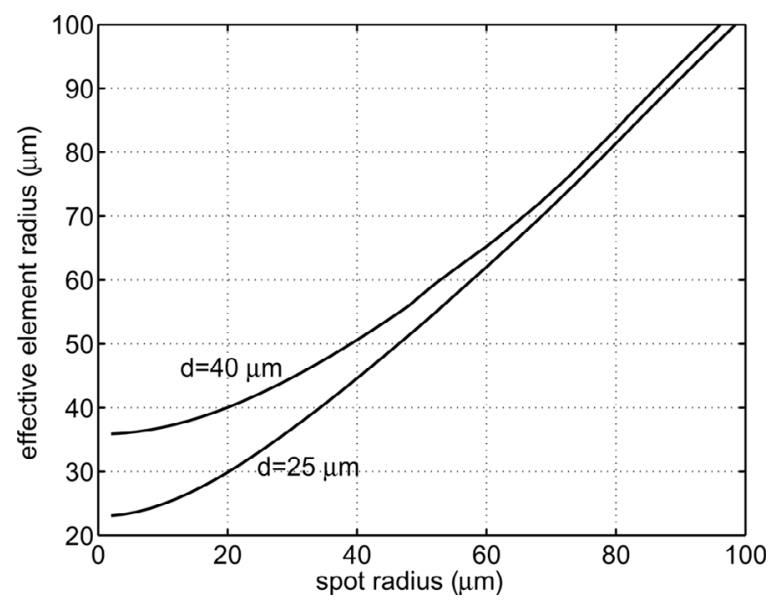

Fig. 10. The effective-element radius, $a_{\text {eff }}$, as a function of the illuminated spot radius, $a$, for a sensor thicknesses $d=40$ and $25 \mu \mathrm{m}$ at a frequency of $25 \mathrm{MHz}$ (polycarbonate-backed sensor). As the illuminated spot radius $a$ is reduced, the effective-element radius eventually reaches a minimum value below which further reductions in $a$ have no effect.

response more noticeably, so that, when $a$ has been reduced to half the sensor thickness $d / 2$, the effective radius $a_{\text {eff }}$ is still twice this size. By reducing $a$ still further, we see that a minimum $a_{\mathrm{eff}}=36 \mu \mathrm{m}$ is reached at $a=d / 4$, $10 \mu \mathrm{m}$, so that any further reductions in $a$ lead to no further reduction in $a_{\text {eff }}$.

In Fig. 10, which shows the effective radius, $a_{\mathrm{eff}}$, as a function of the illuminated spot radius, $a$, for sensor thicknesses of 40 and $25 \mu \mathrm{m}$ and a frequency of $25 \mathrm{MHz}$, this effect can be seen clearly. The effective radius reduces to a minimum below which any further reductions in the illuminated spot radius leave the effective radius unchanged. Fig. 11, which shows the normalized effective-element radius as a function of the normalized wavenumber $k a$ for a number of fixed spot radii, gives a more general result. From Fig. 11, it is clear that, to achieve the absolute minimum, requires both an illuminated spot size less than $d / 4$ and a sufficiently high frequency. At lower frequencies, to the left of the graph, a cosine-type dependence characteristic of a gradient or difference detector dominates the response, and the effective element radius becomes much larger than the illuminated spot radius. We can now see that the examples in Figs. 9 and 10 were all chosen so that the frequency was sufficiently high to ensure the minimum effective radius reached was the absolute minimum achievable.

Three conclusions may be drawn from these graphs:

- for sufficiently large spot sizes and frequencies $(a>2 d$ and $k a \gg 1$ ) the effective element radius equals the spot radius $a_{\text {eff }}=a$,

- it is not possible to achieve an effective element radius smaller than $\sim 0.9 d$ however small the illuminated spot radius, and

- to achieve this minimum, effective-element radius, the illuminated spot radius must be less than $d / 4$, and the frequency sufficiently high, $k d \gtrsim 4$. 


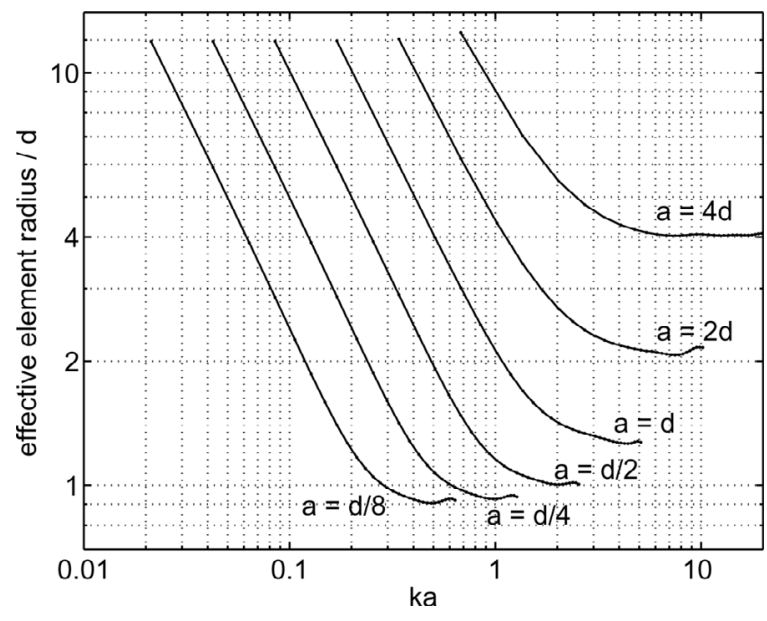

Fig. 11. The effective-element radius, $a_{\text {eff }}$, normalized by the FP layer thickness $d$, as a function of $k a$, the wavenumber normalized by the illumination spot radius, showing that, for $a>2 d$ and $k a \gg 1$, the effective element radius $a_{\text {eff }}=a$; however small the illuminated spot radius, it is not possible to achieve an effective element radius smaller than $\sim 0.9 d$; and to achieve this minimum effective-element radius the actual spot radius must be less than $d / 4$ and the frequency such that $k d \gtrsim 4$ (polycarbonate-backed sensor).

So, for the $40-\mu \mathrm{m}$ thick polymer layer studied here, the minimum attainable, effective-element radius is about $36 \mu \mathrm{m}$, and this can be achieved only with an illumination spot radius of about $10 \mu \mathrm{m}$ and at frequencies above about $20 \mathrm{MHz}$.

\section{Conclusions}

A model of the directivity and frequency response of a planar, FP ultrasound sensor has been described and experimentally validated. Although a three-layer geometry was used here, the model may be straightforwardly extended to sensors with an arbitrary number of layers [19].

Such sensor directivity models have many possible uses. Examples of two applications were given here: the model was used as a design tool to improve the sensor's directional characteristics, and the relationship between actual and effective element radius was investigated. It was found that, for a given sensor thickness, $d$, the minimum achievable, high-frequency, effective-element radius is about $0.9 d$. There are many other potential applications. To improve accuracy in high frequency ultrasonic calibration and metrology, it is becoming increasingly common to take into account the normal-incidence frequency response of the sensor [23], [24]. For sound field characterization, in which the sound waves are not necessarily normally incident, an improvement in accuracy may be achieved by using the model to deconvolve the directional response of the sensor too. In a similar way, deconvolution of the sensor response could reduce artefacts and blurring in imaging applications, in which images are formed by spatially mapping the output of the sensor. As well as removing the effects of the detector response from measurements, the model could be included in a forward model of ultrasonic propagation to predict more accurately real measurements of an ultrasound field [25].

\section{Appendix A}

As stated in Section III-A, a change in the optical phase could be due to a physical change in the thickness of the FP layer or by a change in its refractive index. This second mechanism, whereby a strain causes a change in refractive index, is called the photoelastic effect. In Section III this mechanism was assumed to be negligible, and the optical phase change was modeled as purely due to a change in the thickness. The reasons for making this assumption are discussed in this appendix.

If an acoustic wave traveling through the sensor causes a time-varying stress birefringence in the sensor, the directivity measurements would be expected to depend on the polarization of the interrogating laser beam. Experiments with beams of different polarization showed no significant differences in the directivity measurements. However, although this rules out an anisotropic change in refractive index due to stress birefringence, it does not eliminate the possibility of an isotropic change in the refractive index due to the density changes induced by the wave. This possible transduction mechanism is discussed below, and it also is found to be insignificant for the examples studied here.

In the absence of an acoustic wave, the difference in optical phase between light reflected from the two sides of the interferometer is $\phi=4 \pi n d / \lambda_{0}$ radians. The change in phase due to the two mechanisms, therefore, may be written as:

$$
\Delta \phi=\frac{\partial \phi}{\partial d} \Delta d+\frac{\partial \phi}{\partial n} \Delta n=\frac{4 \pi}{\lambda_{0}}\left(n_{0} \Delta d+d \Delta n\right) .
$$

The second term - due to the photoelastic effect - may be neglected if:

$$
\frac{\Delta n}{n_{0}} \ll \frac{\Delta d}{d} .
$$

Following Pitts and Greenleaf [26], a change in the refractive index of an isotropic, dielectric medium may be related to a small change in the density, $\Delta \rho=\rho-\rho_{0}$, by:

$$
\Delta n=\left(\frac{n_{0}^{2}-1}{2 n_{0}}\right) \frac{\Delta \rho}{\rho_{0}},
$$

where $\rho$ and $\rho_{0}$ are the densities in the presence and absence of a wave, respectively. As the dilatation $\nabla \cdot \mathbf{u}$, the sum of the volumetric strains is related to the density by:

$$
\nabla \cdot \mathbf{u}=\frac{\rho_{0}}{\rho}-1
$$

the change in refractive index can be written:

$$
\Delta n=\left(\frac{n_{0}^{2}-1}{2 n_{0}}\right)\left(\frac{-\nabla \cdot \mathbf{u}}{1+\nabla \cdot \mathbf{u}}\right) .
$$


This change can be calculated using the wave model described in Section III-B. Calculations of the ratio $d \Delta n / n_{0} \Delta d$ showed it be to below 0.001 for the range of frequencies, layer thicknesses, and types of materials discussed here. In addition, this isotropic photoelastic mechanism predicts a frequency response quite different from that measured experimentally [6] and exhibits peaks and troughs in the directional response that disagree with experiment results (Fig. 4).

\section{REFERENCES}

[1] P. C. Beard and T. N. Mills, "An optical fibre sensor for the detection of laser generated ultrasound in arterial tissues," Proc. SPIE, vol. 2331, pp. 112-122, 1994.

[2] V. Wilkens and C. Koch, "Fiber-optic multilayer hydrophone for ultrasonic measurement," Ultrasonics, vol. 37, pp. 45-49, 1999.

[3] Y. Uno and K. Nakamura, "Pressure sensitivity of a fibre-optic microprobe for high frequency ultrasonic field," Jpn. J. Appl. Phys., vol. 38, pp. 3120-3123, 1999.

[4] P. C. Beard, A. M. Hurrell, and T. N. Mills, "Characterization of a polymer film optical fiber hydrophone for use in the range 1 to $20 \mathrm{MHz}$ : A comparison with PVDF needle and membrane hydrophones," IEEE Trans. Ultrason., Ferroelect., Freq. Contr., vol. 47, no. 1, pp. 256-264, 2000.

[5] V. Wilkens, "Characterization of an optical multilayer hydrophone with constant frequency response in the range from 1 to $75 \mathrm{MHz}$," J. Acoust. Soc. Amer., vol. 113, pp. 1431-1438, 2003.

[6] P. C. Beard, F. Perennes, and T. N. Mills, "Transduction mechanisms of the Fabry-Perot polymer film sensing concept for wideband ultrasound detection," IEEE Trans. Ultrason., Ferroelect., Freq. Contr., vol. 46, no. 6, pp. 1575-1582, 1999.

[7] A. Acquafresca, E. Biagi, L. Masotti, and D. Menichelli, "Towards virtual biopsy through an all fiber optic ultrasonic miniaturised transducer: A proposal," IEEE Trans. Ultrason., Ferroelect., Freq. Contr., vol. 50, pp. 1325-1335, 2003.

[8] S. Askenazi, R. Witte, and M. O'Donnell, "High frequency ultrasound imaging using a Fabry-Perot etalon," Proc. SPIE, vol. 5697, pp. 243-250, 2005.

[9] B. T. Cox, E. Z. Zhang, J. G. Laufer, and P. C. Beard, "Fabry Perot polymer film fibre-optic hydrophones and arrays for ultrasound field characterisation," in Adv. Metrol. Ultrasound Med. 2004, J. Physics: Conf. Series, vol. 1, 2004, pp. 32-37.

[10] M. Klann and C. Koch, "Measurement of spatial cross-sections of ultrasound pressure fields by optical scanning means," IEEE Trans. Ultrason., Ferroelect., Freq. Contr., vol. 52, pp. 15461554, 2005.
[11] E. Z. Zhang and P. C. Beard, "Broadband ultrasonic field mapping system using a wavelength tuned, optically scanned focused beam to interrogate a Fabry-Perot polymer film sensor," IEEE Trans. Ultrason., Ferroelect., Freq. Contr., vol. 53, pp. 13301338, 2006.

[12] P. C. Beard, "2D ultrasound receive array using an angle-tuned Fabry Perot polymer film sensor for transducer field characterisation and transmission ultrasound imaging," IEEE Trans. Ultrason., Ferroelect., Freq. Contr., vol. 52, pp. 1002-1012, 2005.

[13] M. Lamont and P. C. Beard, "2D imaging of ultrasound fields using a CCD array to detect the output of a Fabry-Perot polymer film sensor," Electron. Lett., vol. 42, pp. 187-189, 2006.

[14] E. Z. Zhang and P. C. Beard, "2D backward-mode photoacoustic imaging system for NIR (650-1200 nm) spectroscopic biomedical applications," Proc. SPIE, vol. 6086, art. no. 60860H, 2006.

[15] J. M. Vaughan, The Fabry-Perot Interferometer-History, Theory, Practice and Applications. Bristol: Adam Hilger, 1989.

[16] W. Weise, V. Wilkens, and C. Koch, "Frequency response of fiber-optic multilayer hydrophones: Experimental investigation and finite element simulation," IEEE Trans. Ultrason., Ferroelect., Freq. Contr., vol. 49, pp. 937-946, 2002.

[17] L. Brekhovskikh and O. Godin, Acoustics of Layered Media I. Berlin: Springer-Verlag, 1998.

[18] H. Schmidt and F. B. Jensen, "A full wave solution for propagation in multilayered viscoelastic media with application to gaussian beam reflection at fluid-solid interfaces," J. Acoust. Soc. Amer., vol. 77, no. 3, pp. 813-825, 1985.

[19] M. J. S. Lowe, "Matrix techniques for modeling ultrasonic waves in multilayered media," IEEE Trans. Ultrason., Ferroelect., Freq. Contr., vol. 42, pp. 525-542, 1995.

[20] P. C. Beard, "Interrogation of free-space Fabry-Perot sensing interferometers by angle tuning," Meas. Sci. Technol., vol. 14, pp. 1998-2005, 2003.

[21] G. R. Harris, "Hydrophone measurements in diagnostic ultrasound fields," IEEE Trans. Ultrason., Ferroelect., Freq. Contr., vol. 35 , no. 2 , pp. $87-101,1988$.

[22] R. A. Smith, "Are hydrophones of diameter $0.5 \mathrm{~mm}$ small enough to characterise diagnostic ultrasound equipment?," Phys. Med. Biol., vol. 34, no. 11, pp. 1593-1607, 1989.

[23] V. Wilkens and C. Koch, "Amplitude and phase calibration of hydrophones up to $70 \mathrm{MHz}$ using broadband pulse excitation and an optical reference hydrophone," J. Acoust. Soc. Amer., vol. 115, pp. 2892-2903, 2004.

[24] A. Hurrell, "Voltage to pressure conversion: Are you getting 'phased' by the problem?," in Adv. Metrol. Ultrasound Med. 2004, J. Physics: Conf. Series, vol. 1, 2004, pp. 57-62.

[25] B. T. Cox and P. C. Beard, "Fast calculation of pulsed photoacoustic fields in fluids using k-space methods," J. Acoust. Soc. Amer., vol. 117, pp. 3616-3627, 2005.

[26] T. A. Pitts and J. F. Greenleaf, "Three-dimensional optical measurement of instantaneous pressure," J. Acoust. Soc. Amer., vol. 108, no. 6, pp. 2873-2883, 2000. 